\title{
The virial balance of clumps and cores in molecular clouds
}

\author{
Sami Dib ${ }^{1}$, Enrique Vázquez-Semadeni ${ }^{1}$, Jongsoo Kim ${ }^{2}$, \\ Andreas Burkert ${ }^{3}$ and Mohsen Shadmehri ${ }^{4}$ \\ ${ }^{1}$ Centro de Radioastronomía y Astrofísica, UNAM, Apdo. 72-3 (Xangari), 58089 Morelia, \\ Michoacán, Mexico \\ email: s.dib@astrosmo.unam.mx \\ ${ }^{2}$ Korea Astronomy and Space Science Institute, 61-1, Hwaam-dong, Yuseong-gu, Daejeon \\ 305-764, Korea \\ ${ }^{3}$ University Observatory Munich, Scheinerstrasse 1, D-81679 Munich, Germany \\ ${ }^{4}$ Department of Physics, School of Science, Ferdowsi University, Mashhad, Iran
}

\begin{abstract}
We study (i.e., Dib et al. 2006) the virial balance of clumps and cores (CCs) in a set of three-dimensional numerical simulations of driven, magnetohydrodynamical, isothermal molecular clouds (MCs). The simulations represent a range of magnetic field strengths in MCs from subcritical to non-magnetic regimes. We developed a clump-finding algorithm to identify CCs at different threshold levels in the simulation box, and for each object, we calculate all the terms that enter the virial theorem in its Eulerian form. We also calculate, other quantities commonly used to indicate the state of gravitational boundedness of CCs such as the Jeans number $J_{c}$, the mass-to magnetic flux ratio $\mu_{c}$, and the virial parameter $\alpha_{v i r}$. Our results suggest that a) CCs are dynamical out-of-equilibrium structures. b) The surface energies are of the same order than their volume counterparts and thus are very important in determining the exact energy balance in CCs. c) CCs can be either in the process of being compressed by the velocity field or of being dispersed. Yet, not all CCs that have a compressive velocity field at their boundaries are necessarily gravitationally bound. d) There is no one-to-one correspondence between the state of gravitational boundedness of a CC as described by the energy balance analysis (i.e., gravity versus other energies) or as implied by the classical indicators $J_{c}, \mu_{c}$, and $\alpha_{v i r}$. In general, from the energy analysis, we observe that only the inner regions of the objects (i.e., the dense cores selected at high threshold levels) are gravitationally bound, whereas $J_{c}$ and $\alpha_{v i r}$ estimates tend to show that they are more gravitationally bound at the lowest threshold levels. g) We observe, in the non-magnetic simulation, the existence of a bound core with structural and dynamical properties that resemble those of the Bok globule Barnard 68 (B68). This suggests that B68 like cores can form in a larger molecular cloud and then be confined by the warm gas of a newly formed HII region, which can heat and rarefy the gas around the core, confine it, and extend its lifetime.
\end{abstract}

Keywords. ISM: structure, kinematics and dynamics, magnetic fields, clouds, globules, individual (Barnard 68)

\section{Acknowledgements}

S. D. is supported by a UNAM postdoctoral fellowship and is grateful to the Symposium organizers, Profs Jan Palouš and Bruce Elmegreen for the financial support that allowed him to attend the conference.

\section{Reference}

Dib, S., Vázquez-Semadeni, E., Kim, J., Burkert, A., \& Shadmehri, M. 2006, ApJ submitted, (astro-ph/0607362) 